\title{
Obstáculos a la actividad exportadora de las empresas del Perú
}

$\begin{array}{r}\text { Oscar Malca Guaylupo } \\ \text { omalca@up.edu.pe } \\ \text { Universidad del Pacífico. Lima. Perú } \\ \text { Jorge Rubio Donet } \\ \text { rubio_jl@up.edu.pe } \\ \text { Universidad del Pacífico. Lima. Perú } \\ \hline\end{array}$

\section{Resumen}

El presente artículo plantea el estudio del comportamiento de las empresas exportadoras peruanas como base para el análisis del desarrollo del sector exportador del país. Para ello se centra en los obstáculos a la exportación, a partir de una identificación y clasificación de los mismos que sirva a su vez de referencia para posteriores estudios. Por otra parte se lleva a cabo un estudio empírico para la identificación de dichos obstáculos en el caso de las empresas peruanas.

\section{Palabras clave:}

Internacionalización, empresa peruana, exportaciones, mercados internacionales, desempeño exportador, 


\section{Introducción}

La expansión de las exportaciones de un país, por lo general, tiene efectos positivos en el crecimiento de la economía y en el de las empresas individuales (Cavusgil \& Nevin, 1981). Asimismo, la exportación es de vital importancia económica para las naciones comerciantes y sus empresas ya que aumenta la rentabilidad, mejora la utilización de la capacidad, crea empleo y mejora la balanza comercial (Kaynak \& Barker, 1992; Abassi, Shakhsian, Fayyazi, \& Rezaie, 2012).

Sin embargo, a pesar de los numerosos beneficios que trae consigo la exportación, la mayoría de las empresas no exportan, a pesar que se considera a la exportación como inevitable en los mercados mundiales cada vez más integrados (Pinho \& Martins, 2010; Milanzi, 2012).

Asimismo, la internacionalización se ha convertido en una ruta para el crecimiento de las empresas que buscan expandir sus operaciones y el caso de las barreras de exportación es de especial interés debido a que la exportación es el modo de entrada más utilizada para el mercado internacional (Leonidou, 1995).

En su gran mayoría, los obstáculos explican el porqué de la existencia de empresas que no exportan, que exportan de manera esporádica o que no aprovechan todo su potencial exportador. Por ello, la minimización o eliminación de estas contribuirá con un mayor rendimiento exportador que beneficiará eventualmente a las empresas y a sus respectivos países (Bilkey, 1978).

Por estas razones, son diversas las investigaciones que se vienen desarrollando con el objeto de identificar y superar las diferentes barreras a la exportación (Leonidou, 1995b). De acuerdo con la literatura, superar las barreras es uno de los factores más eficaces que contribuye a mejorar el desempeño de las exportaciones de cualquier empresa, que a su vez, puede conducir a la mejora de los índices de rendimiento (Abassi, Shakhsian, Fayyazi, \& Rezaie, 2012).

En el caso peruano, durante la última década (2002-2012), las exportaciones peruanas casi han sextuplicado su valor de US\$ 7,713 a US\$ 45,933 millones, llegando a representar el 26 por ciento del PIB, pero con un ligero estancamiento para el periodo 2011-2012. Hacer sostenible el crecimiento exportador, implica conocer el comportamiento de la empresa exportadora peruana con el objeto de conocer las causas de su desempeño, por eso es necesario desarrollar investigaciones que permitan analizar y fomentar el debate a efecto de contribuir con las mejoras, tanto a la gestión de la empresa peruana, como a los diferentes programas de promoción de exportaciones que el Estado peruano desarrolla.

De acuerdo con Malca y Rubio (2013), se identifican tres tipos de empresas que participan en las exportaciones peruanas: exportadoras continuas (que tienen tres 0 más años de exportación continua), empresas esporádicas (aquellas que no tienen continuidad en su evolución exportadora) y exportadores únicos (empresas que exportaron solamente una vez). De esta clasificación, las empresas esporádicas representan un porcentaje muy elevado que dependiendo del sector fluctúan entre el $87 \%$ y el $94 \%$ del total de las empresas que exportan y muestran una alta probabilidad de convertirse en exportadoras. 
Esta clasificación, concuerda con lo desarrollado por Leonidou (2004) quien clasifica a las empresas como: (1) no exportadoras, (2) exportadoras y (3) ex exportadoras. Asimismo, los estudios muestran que el impacto de las barreras de exportación varía entre los tres grupos de empresas, utilizando un tratamiento diferente para cada una de ellas por parte de los programas de promoción de exportaciones (Keng, Kau Ah \& Tan Soo Jiuan, 1989; Yaprak, 1985; Tesar \& Jesse, 1982; Leonidou, 2004).

Por lo mencionado, y por el alto volumen de empresas esporádicas y la probabilidad de que éstas se conviertan en exportadoras continuas es importante, para el desarrollo exportador del Perú, identificar los principales obstáculos que limitan la actividad exportadora de las empresas esporádicas.

Los objetivos del presente artículo son dos: primero, el desarrollo del sector exportador del país, desde diversos enfoques necesita un constante estudio del comportamiento de las empresas exportadoras peruanas, como es el caso de los obstáculos a la exportación, por lo que es necesario realizar una investigación bibliográfica que nos permita identificar y clasificar los diferentes obstáculos a la exportación y sirva de referencia para posteriores estudios. Segundo identificar los principales obstáculos a la actividad exportadora, a través de la aplicación de un estudio empírico.

\section{Marco Conceptual}

Durante las tres últimas décadas, se han realizado una gran cantidad de investigaciones en todo el mundo, con el objetivo de conocer cuáles son los factores que impiden la puesta en marcha, el desarrollo y la sostenibilidad de las operaciones de exportación de una empresa. Los primeros estudios aparecieron por primera vez en la década de 1960, pero el impulso de la investigación se produjo en la década de 1980 y principios de 1990 (Leonidou, 1995).

Leonidou L.C. (1995) indica que Alexandrides (1971) fue el primero en investigar sobre las barreras de la exportación. Su investigación mostró que algunos de los mayores problemas que limitan a que las empresas comiencen a exportar son la fuerte competencia que existe en el extranjero, la falta de conocimiento para exportar, la comprensión insuficiente de los procedimientos y las dificultades de localizar mercados extranjeros (Alexandrides, 1971; Leonidou, 1995).

Bilkey \& Tesar (1977) desarrollaron un modelo de seis etapas para explicar el desarrollo del proceso exportador de la empresa y, a través de estas, identificaron que las percepciones de las barreras a la exportación varían según la etapa en que se encuentre la empresa. Los autores concluyen que mientras más avanzada se encuentre la empresa en la etapa de exportación, más alto será el porcentaje de empresas que perciban las dificultades para entender las prácticas empresariales en el extranjero, las diferentes especificaciones de los productos y estándares de consumo, problemas derivados de la cobranza de las exportaciones, así como obtener una adecuada representación en los mercados extranjeros. Por otro lado, obtener los fondos necesarios para iniciar las operaciones de exportación fue difícil para las empresas en las primeras etapas de la exportación. (BILKEY \& TESAR, 1977) (Leonidou L. C., Export Barriers: Non-exporters' Perceptions, 1995). 
Tesar and Tarleton (1982) indican que las empresas están más preocupadas por identificar oportunidades en el extranjero que por la misma exportación, ya que una vez que esta se lleve a cabo, la prioridad se centra en la búsqueda de una representación adecuada en el mercado internacional. (Tesar and Tarleton, 1982; Leonidou, 1995).

Según Leonidou L.C. (1995), Kaynak and Kothari (1983) fueron los primeros en introducir el análisis transcultural en la investigación de barreras de la exportación. En su estudio, encontraron varias diferencias entre los no exportadores de las regiones de Scotia y Texas en cuanto a sus percepciones de los impedimentos a las exportaciones. Por otro lado, su análisis mostró que las diferencias entre los no exportadores y exportadores difieren en los diferentes países (Kaynak and Kothari, 1983; Leonidou, 1995). Barrett and Wilkinson (1985) encontraron que los obstáculos particularmente importantes son la falta de capacidad para encontrar precios competitivos de proveedores en el extranjero y los altos costos del transporte internacional (Barret and Wilkinson, 1985; Leonidou, 1995).

Por otra parte cabe hablar de las barrearas a la exportación. Las barreras a la exportación son todos aquellos obstáculos, limitaciones actitudinales, estructurales, operativas y otras que disuade o impiden la capacidad para iniciar, desarrollar o mantener las operaciones comerciales en los mercados extranjeros. (Leonidou, 1995; Leonidou, 2004).

A su vez, las barreras pueden ser clasificadas en internas y externas (Leonidou, 2004). Las internas están asociadas a la insuficiencia de los recursos y capacidades organizacionales de la empresa. (Tesfom \& Lutz, 2006), mientras que las barreras externas son las que se derivan del ambiente en donde la empresa va a operar; es decir, tanto del mercado de exportación o del entorno nacional. (Leonidou, 2004; Leonidou, 1995).

Por otro lado, se pueden identificar tres grupos de empresas: (1) no exportadoras, (2) exportadoras y (3) ex exportadoras, por lo que el impacto de las barreras de exportación varía entre los tres grupos de empresas, utilizando un tratamiento diferente para cada una de ellas por parte de los programas de promoción de exportaciones (Keng, Kau Ah, and Tan Soo Jiuan 1989; Yaprak, 1985, Tesar and Jesse 1982; Leonidou, 2004).

También en la literatura podemos identificar de manera clara que los países desarrollados son los que constantemente se preocupan por la identificación, análisis y propuestas para superar las barreras que limitan un mejor desempeño de sus empresas en el mercado de exportación. En la tabla 1 se muestra el listado/clasificación de estudios por países: 
Tabla 1: Listado/Clasificación de países - № de estudios

\begin{tabular}{|l|l|}
\hline Estados Unidos \\
\hline Gran Bretaña \\
\hline Australia \\
\hline Brasil \\
Singapur \\
Chipre \\
\hline Turquía \\
\hline Dinamarca
\end{tabular}

13

7

4

3

3

3

2

2

2

Noruega

Corea del Sur

Canadá

Grecia

2

2

Arabia Saudita

2

España

Alemania

Taiwan

Lituania

Nueva Zelanda

Vietnam

Eritrea

Portugal

Estudios comparativos, dos o más países.

6

Total

En las tablas 2 y 3 se muestra una clasificación general de los aspectos internos y externos que se configuran como barreras a la exportación. 
Tabla 2: Barreras internas a la exportación

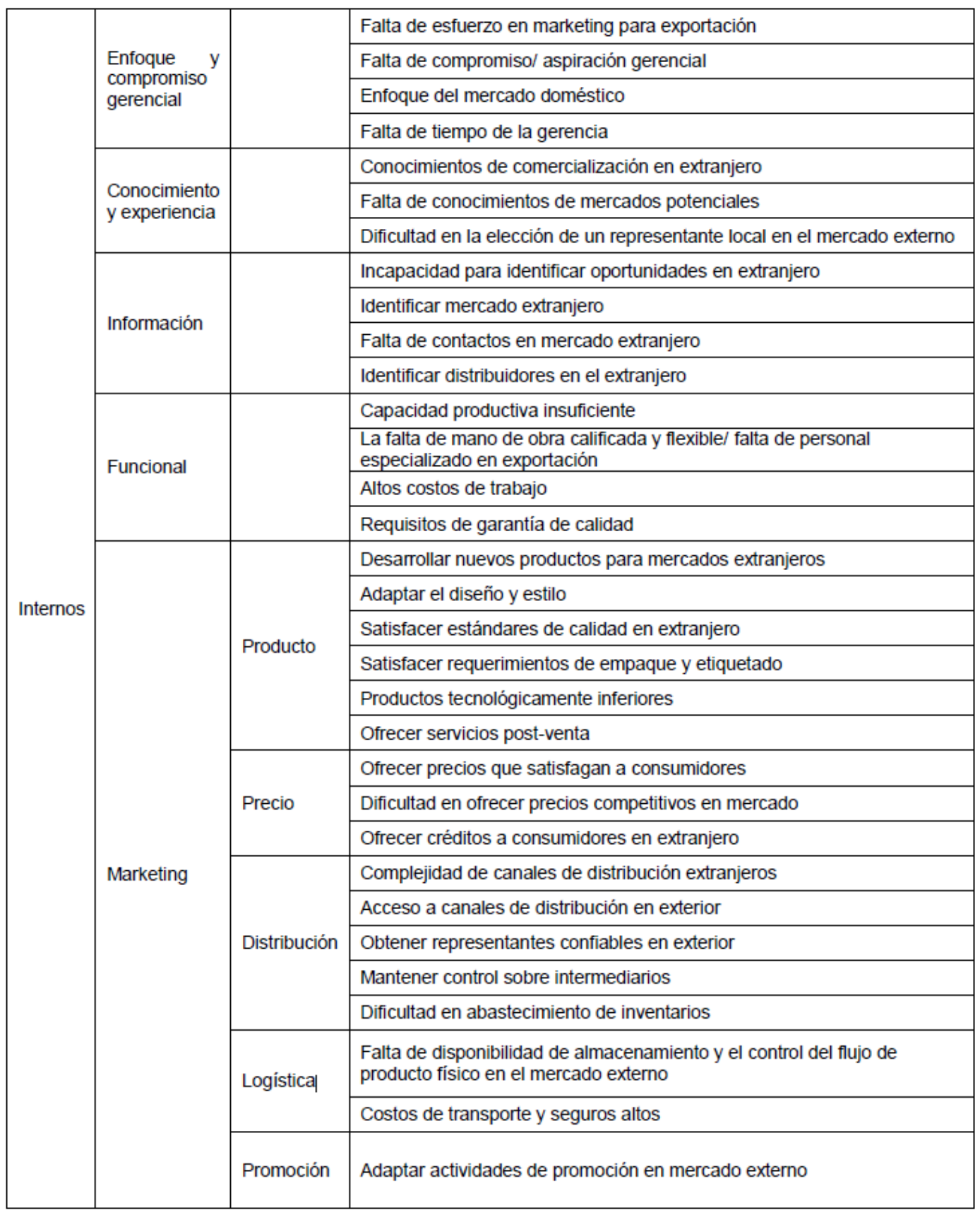


Tabla 3: Barreras externas a la expotación

\begin{tabular}{|c|c|c|c|}
\hline \multirow{29}{*}{ Externos } & \multirow{23}{*}{$\begin{array}{l}\text { Mercado } \\
\text { de } \\
\text { destino }\end{array}$} & \multirow{5}{*}{ Económicas } & Fuerte posición de moneda doméstica \\
\hline & & & Minimizar fluctuaciones del tipo de cambio \\
\hline & & & Tasa de interés e inflación \\
\hline & & & Deterioro de condiciones económicas en mercado externo \\
\hline & & & Riesgos cambiarios de moneda extranjera \\
\hline & & \multirow{3}{*}{$\begin{array}{l}\text { Político } \\
\text { Legal }\end{array}$} & Inestabilidad política \\
\hline & & & Desconocimiento de leyes en mercado extranjero \\
\hline & & & Requerimientos burocráticos \\
\hline & & \multirow{4}{*}{ Sociocultural } & Diferencias en lenguaje verbal y no verbal \\
\hline & & & Diferentes hábitos y actitudes de clientes extranjeros \\
\hline & & & Rasgos socioculturales diferentes \\
\hline & & & Formas de negociación en extranjero \\
\hline & & \multirow{3}{*}{$\begin{array}{l}\text { Restricciones } \\
\text { y } \\
\text { regulaciones } \\
\text { en mercado } \\
\text { exterior }\end{array}$} & Barreras arancelarias \\
\hline & & & Barreras no arancelarias \\
\hline & & & Falta de asesoramiento financiero \\
\hline & & \multirow{8}{*}{$\begin{array}{l}\text { Obstáculos al } \\
\text { desarrollo del } \\
\text { mercado }\end{array}$} & Costos de desarrollo de mercado \\
\hline & & & Falta de financiamiento de capital \\
\hline & & & Competencia en mercado exterior \\
\hline & & & Corrupción en mercado objetivo \\
\hline & & & Recolección y transferencia de fondos \\
\hline & & & Costos de transporte y distribución \\
\hline & & & Manejo de documentación de exportación \\
\hline & & & Recolección lenta de pagos en el extranjero \\
\hline & \multirow{6}{*}{$\begin{array}{l}\text { Mercado } \\
\text { de } \\
\text { origen }\end{array}$} & \multirow{4}{*}{$\begin{array}{l}\text { Políticas de } \\
\text { Gobierno }\end{array}$} & Falta de apoyo del gobierno \\
\hline & & & Políticas de gobierno inconsistentes \\
\hline & & & Restricciones y regulaciones del gobierno \\
\hline & & & Falta de incentivos a la exportación \\
\hline & & \multirow{2}{*}{$\begin{array}{l}\text { Riesgo } \\
\text { retornos }\end{array}$} & $\begin{array}{l}\text { Bajas expectativas de retomo/ baja percepción de rentabilidad de } \\
\text { exportaciones }\end{array}$ \\
\hline & & & Alto riesgo percibido por operaciones en extranjero \\
\hline
\end{tabular}

De acuerdo con la revisión de la literatura, podemos ver que son numerosas y diversas las barreras a la exportación, en parte esta gran cantidad de variables se debe a la naturaleza de los entornos que cada empresa tiene y a las diferentes percepciones que los empresarios tienen de ellas. Por ello, se proponen las siguientes hipótesis principales:

H1. El desconocimiento tanto de los mecanismos de acceso a los mercados de exportación, así como de los diferentes procesos relacionados a la investigación de mercados y de las operaciones de exportación, afecta negativamente a las decisiones de mantenerse en los mercados internacionales.

H2. Las restricciones de los recursos internos de la empresa afectan al cumplimiento de los objetivos de exportación de la empresa.

H3. La inestabilidad social, política y económica del mercado de destino afecta al proceso de exportación. 


\section{Metodología}

Con el propósito de alcanzar los objetivos del trabajo, se utilizó como herramienta de medición una encuesta, la cual fue aplicada a través de entrevistas a funcionarios de las empresas esporádicas. Para el desarrollo de la encuesta, se tuvo en cuenta la revisión de la literatura que permitió identificar y analizar las diferentes variables, así como comparar las encuestas desarrolladas por diversos autores como Paulo da Silva, (2001), (Ortiz 2003), Artega-Ortiz \& Fernandez-Ortiz (2010) y Okpara (2010); las mismas que fueron validadas en diferentes investigaciones. En todos los casos los descriptores alcanzaron una medida de fiabilidad Alfa de Cronbach superior a 0.7. El Alfa de Cronbach indica que los ítems (medidos en escala tipo Likert) evalúan un mismo constructo y que están altamente correlacionados (Hair, 1999), de modo que se tenga una alta confianza en la herramienta de medición propuesta. Asimismo, se tuvo reuniones de trabajo con funcionarios del área de Información y Negocios Electrónicos de PROMPERU, a cargo del Coordinador Carlos Díaz. También, se contó con el apoyo de la Gerencia de Comercio Exterior de la Cámara de Comercio de Lima a cargo de Carlos García, con quienes los autores del presente trabajo tuvimos diversas reuniones para analizar la encuesta. Finalmente, se optó por adoptar la encuesta de Arteaga-Ortiz \& Fernandez-Ortiz (2010), que se presenta en el anexo 1.

Las razones que tuvimos para escoger la encuesta indicada son varias, como, ser una encuesta desarrollada sobre la base de la revisión de los principales estudios teóricos y empíricos, para proponer una clasificación integradora. Asimismo, realizó una comparación empírica de las percepciones, por lo que la clasificación puede ser universalmente aceptada y utilizada en futuros estudios sobre las exportaciones. Igualmente, para validar su propuesta utilizó la metodología de ecuaciones estructurales, más conocida como "SEM" (por sus siglas en inglés Structural Equation Modeling) que en la actualidad es la modelización más avanzada, dado que SEM combina las técnicas del análisis multivariante (Cepeda Carrión \& Roldán Salgeiro, 2004) y cuenta con pruebas estadísticas de bondad de ajuste para el factor propuesto. (Artega-Ortiz \& Fernandez-Ortiz, 2010), Además, las dimensiones propuestas ofrecieron un alto valor explicativo de la variable exógena y confirma tanto la fiabilidad de la escala como la validación de los constructos, utilizando, observaciones que fueron corroboradas estadísticamente.

Por otro lado, una vez que se tuvo la encuesta, esta fue analizada tanto con los funcionarios de PROMPERU como de la Cámara de Comercio y se acordó incluir dos preguntas adicionales abiertas para que los entrevistados incluyan y califiquen otras barreras que no se encontraran en el cuestionario.

Las preguntas de la encuesta de Arteaga - Ortiz \& Fernandez - Ortiz (2010) están clasificadas en cuatro grupos de variables: barreras de conocimiento, que corresponden a las preguntas P1 a P6; barreras de recursos, que corresponden a las preguntas $\mathrm{P} 7$ a $\mathrm{P} 11$; barreras de procedimiento, que corresponden a las preguntas P12 a P21; barreras exógenas, que corresponden a las preguntas P22 a P26. Esta clasificación se realizó con el fin de reducir la gran dispersión de variables que resultaron de la revisión teórica. 
Dado que la encuesta original se encontraba en el idioma inglés, los autores realizaron la traducción la misma que fue discutida con los funcionarios de las entidades indicadas. También, fue probada tanto con funcionarios especializados en comercio internacional de la Cámara de Comercio de Lima, así como con gerentes de empresas elegidas por conveniencia.

Para la recolección de datos se entrevistó a los gerentes de las empresas que tuvieron una actividad exportadora durante los 10 últimos años. Considerando que en el marco muestral incluye tanto a empresas con continuidad exportadora como a empresas esporádicas es conveniente señalar que el presente estudio debe ser considerado como un estudio exploratorio de los obstáculos en la actividad exportadora, focalizado en las empresas esporádicas.

La principal limitación para el desarrollo del estudio fue la ubicación de las empresas esporádicas, dado que los datos que aparecen en Sunat-Aduanas no concuerdan con la realidad. Asimismo, la persona a ser entrevistada por diversas razones no era ubicable. Los métodos empleados para la entrevista fueron: el uso de una encuestas online mediante Google-docs, asistencia a eventos de empresarios exportadores, llamadas telefónicas, entrevistas personales.

De estos cuatro métodos, lo que mejor funcionó fue una combinación de los dos últimos. Es relevante indicar que la tasa de respuesta online fue excesivamente baja, a tal extremo que la cifra no es relevante. Debemos hacer público nuestro agradecimiento a los alumnos de la Facultad de Ciencias Empresariales de la Universidad del Pacífico, así como a los funcionarios de PROMPERU - Exportaciones, a la Cámara de Comercio de Lima-CCL y de la Asociación de Exportadores - ADEX, quienes gentilmente nos apoyaron difundiendo la encuesta entre sus asociados para poder obtener citas y entrevistar a los empresarios, y de esta manera hacer realidad este primer estudio de los obstáculos a la actividad exportadora.

Para el estudio se aplicó a la información recolectada mediante una muestra inicial de 80 empresas que desarrollaron una actividad exportadores en el último año. En la encuesta se incluyeron un total de 26 preguntas Likert, con valores entre 1 y 7 , para medir la percepción de los empresarios acerca de las barreras que limitan la actividad exportadora. Con los datos de las encuestas se encontró una media aproximada de 4.2, con una variancia de 3.8. Teniendo como base estos resultados y si se establece un nivel de precisión de 0.5 en la estimación de la calificación promedio, con una confianza del $95 \%$ se tiene que el tamaño de muestra requerido es de 80 encuestas, aproximadamente.

$$
n=\frac{T_{(1-0.05 / 2,80-1)}^{2} S^{2}}{d^{2}} \cong \frac{\left(2^{2}\right)(3.8)}{(0.4)^{2}} \cong 95
$$

Considerando que el número de empresas que desarrollaron una actividad exportadora en el 2013 fue mayor que 2000, luego, el tamaño mínimo de muestra requerido para esta investigación resulta ser de 95 encuestas (Lohr, 2000). Para la recolección de datos se aplicó una muestra irrestricta de tamaño 150 entre las empresas exportadoras durante el 2013, según la información proporcionada por 
Promperu. Del total de encuestas programadas, se recolectó un total de 120 encuestas, no obstante se eliminó una de ellas por tener datos incompletos.

Teniendo en cuenta los objetivos del estudio y de las hipótesis de trabajo planteadas en la presente investigación, para realizar el análisis estadístico se propone utilizar dos herramientas estadísticas multivariadas: el análisis factorial, y el análisis de escalamiento multidimensional.

El análisis factorial tiene como objetivo explicar la estructura causal que origina las relaciones entre un conjunto de variables, así como la variación específica de cada una de ellas (Anderson, 1999). Este análisis se propone con el propósito de dar respuesta a la pregunta: ¿Qué barreras a la exportación son las que tienen mayor influencia en la actividad exportadora de las empresas?

Se pretende construir un modelo que explique la correlación existente entre las barreras a la exportación y los principales factores. El objetivo consiste en seleccionar un conjunto de factores que expliquen las interrelaciones entre los factores que limitan las actividades exportadoras.

Mediante el análisis factorial se pretende construir un modelo para explicar las correlaciones existentes entre los factores que influyen en la actividad exportadora, y expresarlos en términos de otro conjunto de menor cantidad de variables denominadas factores (Cuadras, 2012; Dallas, 1998; Ledolter, 2013).

La estimación de los parámetros del modelo factorial implica el uso de métodos que derivan en lo que se denomina la extracción de factores, determinando así el número de factores comunes a ser seleccionados en el análisis factorial. Teniendo en cuenta que la información primaria a utilizar está basada en escalas Likert (Kinnear, 1996), se utilizará el método de estimación de los componentes principales (método exploratorio), en la determinación de la existencia y número de los factores.

En el caso del análisis de los factores que influyen en la actividad exportadora, básicamente el interés consiste en investigar qué factores comunes dominan la actividad exportadora y que factores de exportación tienen un componente específico importante. Para una interpretación más sencilla de las cargas factoriales se suele realizar una rotación de los ejes factoriales. Varios métodos han sido sugeridos para realizar este proceso (Cuadras, 2012; Lehmann, 1997; Hair, 1999).

El escalamiento multidimensional involucra a un conjunto de técnicas que permiten la elaboración de una representación espacial de un conjunto de objetos, teniendo en cuenta un conjunto de mediciones sobre las percepciones o preferencias de individuos. Uno de los objetivos es construir mapas perceptuales de pocas dimensiones, teniendo en cuenta las proximidades existentes entre un conjunto de mediciones Likert referidas a un grupo de factores o variables (Cuadras, 2012; Lehmann, 1997; Dallas, 1998).

El escalamiento multidimensional constituye una técnica alternativa al análisis factorial, y que tiene la ventaja de que no requiere que las mediciones tengan una escala de medición alta. De modo similar en la búsqueda de las relaciones existentes entre un conjunto de factores, es posible mediante el análisis de escalamiento multidimensional 
deducir nuevas dimensiones subyacentes (Hair,1999), a partir de una serie de juicios de preferencia o similitud realizados por los encuestados sobre los factores que limitan a la actividad exportadora.

De manera similar a los casos anteriores, al trabajar con escalas no métricas (escalas Likert (Pagano, 1999)), se debe realizar procesos de estimación no paramétrico. Para la estimación de los parámetros se utilizará el método de estimación de los componentes principales, y la representación será obtenida sobre la base de los dos primeros componentes (Ledolter, 2013).

\section{Datos}

Las 119 empresas entrevistadas pertenecen a diferentes sectores, siendo hegemónicas las empresas del sector agrícola (agroexportación y agroindustria), las cuales concentran el $45.22 \%$, y las de textiles y confecciones que representan el $20.87 \%$. En la tabla 4 se puede ver la composición porcentual de las empresas según los sectores:

Tabla 4: Empresas según sectores

\begin{tabular}{lr}
\hline Sector & $\%$ \\
\hline Agroexportadora & 42.61 \\
Agroindustria & 2.61 \\
Industrial & 5.22 \\
Metal mecánica & 3.48 \\
Otros & 7.83 \\
Pesca & 6.09 \\
Químico & 0.87 \\
Servicios & 10.43 \\
Textil y confecciones & 20.87 \\
\hline Total & 100 \\
\hline
\end{tabular}

Respecto a los entrevistados: el $33.61 \%$ son gerentes generales, el $38.66 \%$ son gerentes de áreas funcionales y el $27.73 \%$ ocupan otros cargos; respecto a la edad de los mismos el $8 \%$ tienen menos de 30 años, el $26 \%$ entre 31 y 40 , el $21 \%$ entre 41 y 50 , el $25 \%$ tiene más de 50 y el $20 \%$ no poseen edad identificada; respecto a la formación el 2\% cuenta con MBA, el 2\% con posgrado, el 93\% con formación universitaria y el $3 \%$ con formación técnica; respecto al género el $71 \%$ son hombres y el $29 \%$ mujeres. 


\section{Resultados}

Respecto a la adecuación de los datos, puesto que las variables en estudio corresponden a escalas Likert, cada una de ellas con valores entre 1 y 7 , no fue necesario realizar un proceso de estandarización u otra transformación.

Antes de iniciar el análisis de los datos se llevó a cabo un análisis para evaluar la consistencia de las respuestas. Un estudio sobre las correlaciones entre las variables se encontró:

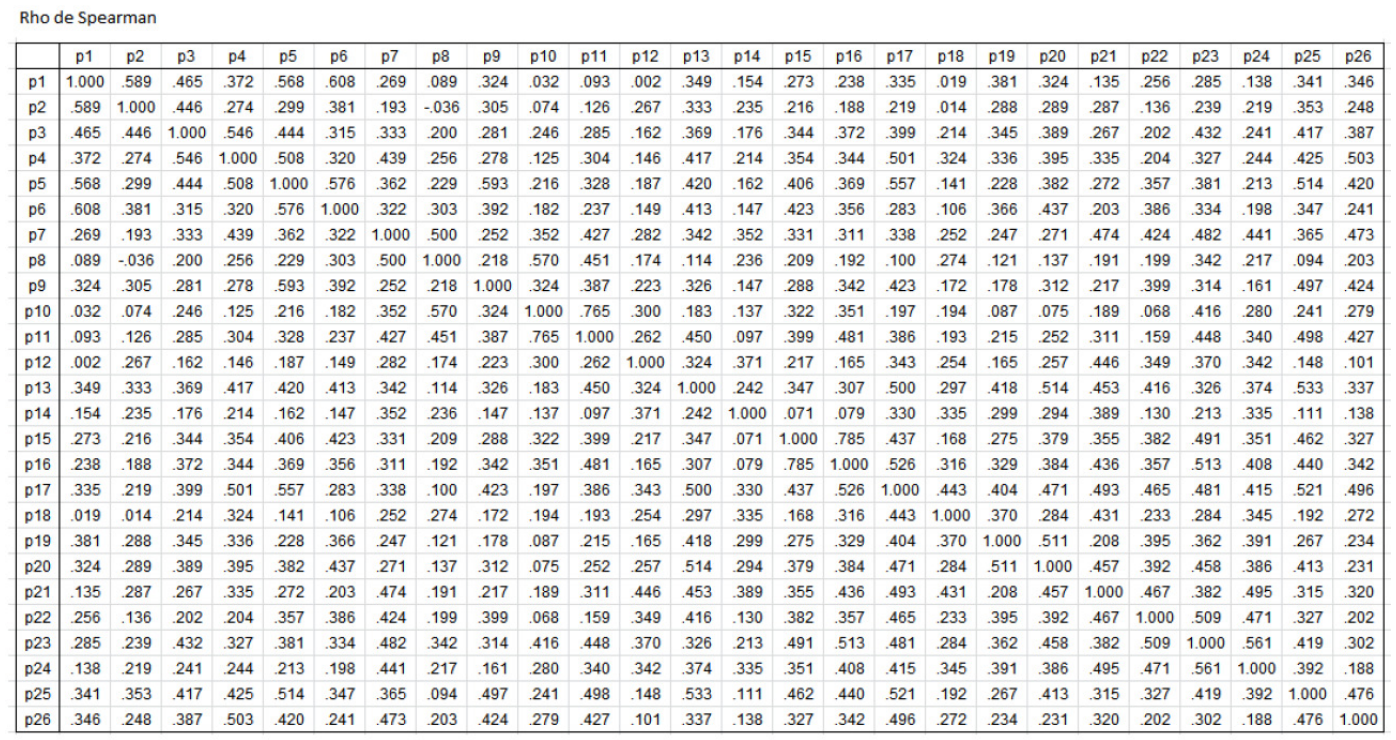

Las correlaciones de Spearman fueron todas positivas lo que significa que todas las variables de la encuesta están interactuando en el mismo sentido, y que por tanto en un proceso de acumulación no se produce un efecto contradictorio en la agregación, y además siendo gran parte de estas correlaciones significativas se justifica la aplicación del análisis factorial y escalamiento multidimensional mencionados anteriormente.

Por otro lado, el gráfico de cajas que se muestra a continuación se aprecia que en ningún caso se tiene respuestas atípicas que podría estar afectando a las tendencias generales. En todas las variables se observa respuestas con un comportamiento con ligeras asimetrías a la izquierda; es decir, para todas las variables se observa que hay empresarios que dieron calificaciones por debajo a la tendencia usual de la calificación general. 


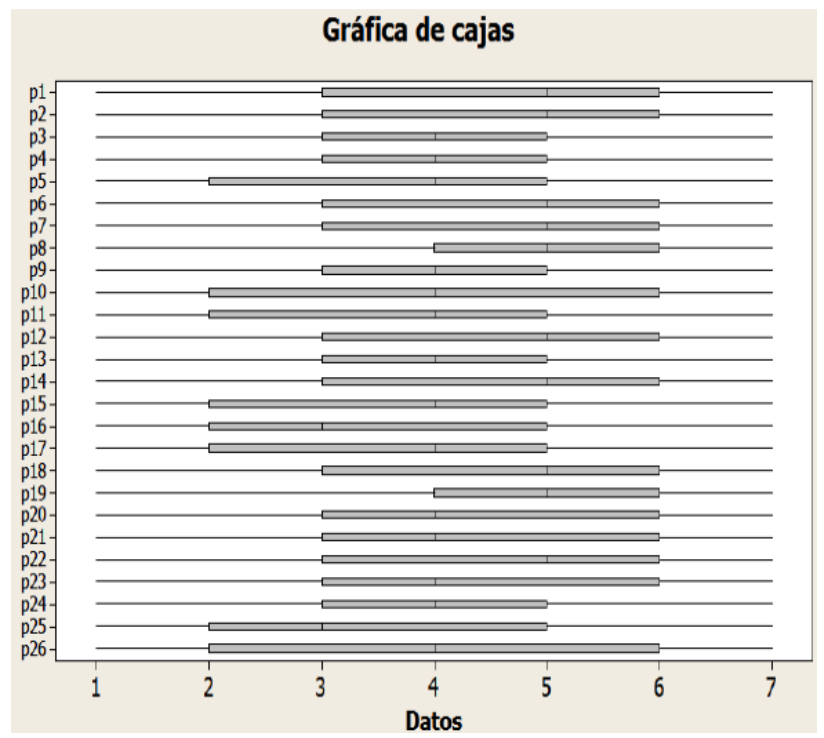

Para evaluar la consistencia de las respuestas se utilizó el coeficiente Alfa de Cronbach (Pagano, 1999; Lehmann, 1997), cuyo resultado fue ,926 indicando que existe una gran consistencia y confiabilidad en los datos recolectados, y que además los indicadores propuestos en la investigación muestran una alta consistencia y fiabilidad de los instrumentos de medición.

Siendo los datos mediciones Likert obtenidas con un mismo criterio, no fue necesario hacer una estandarización para realizar el análisis. Al obtener los reportes del análisis factorial se encuentra que:

KMO y prueba de Bartlett
\begin{tabular}{|ll|r|}
\hline Medida de adecuación muestral de Kaiser-Meyer- &, 840 \\
Olkin. & & 1714,000 \\
Prueba de esfericidad de & Chi-cuadrado & \\
Bartlett & aproximado & 325 \\
& gl &, 000 \\
\hline
\end{tabular}

La prueba de Bartlett resulta significativa, lo que indica que los factores estudiados están fuertemente correlacionados y que por tanto es justificable un análisis factorial. De otro lado, el coeficiente KMO de 0.84 muestra que existe una alta adecuación de los datos al modelo factorial. 


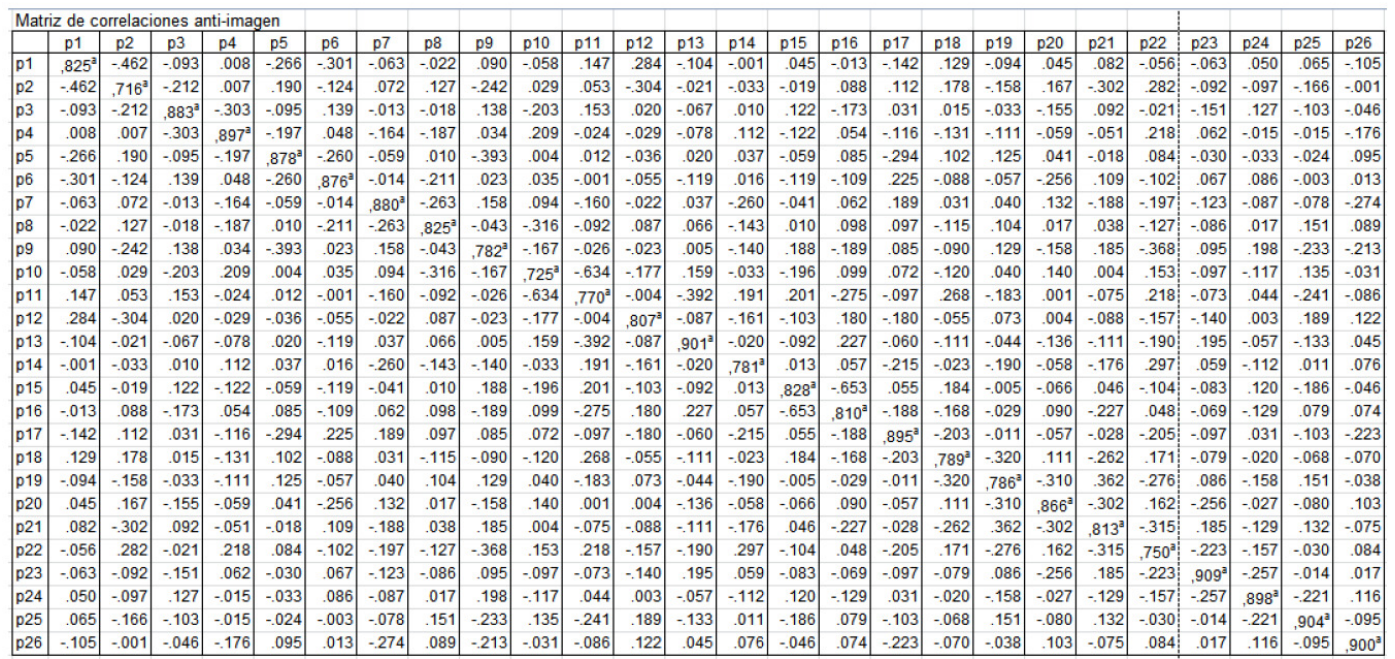

Los coeficientes MSA de las variables se encuentran en la diagonal de esta matriz. Todos factores incluidos en el análisis tienen un coeficiente MSA mayor que 0.5 , lo cual quiere decir que son factores que deben ser incluidos en el análisis factorial.

Las variancias explicadas por los principales factores se muestran en el siguiente reporte. Se puede apreciar que el primer factor explica por si solo un $35.8 \%$ de la variación total, y que luego la explicación individual de los otros factores cae significativamente.

\begin{tabular}{|c|c|c|c|c|c|c|}
\hline \multirow[b]{3}{*}{ Componente } & \multicolumn{6}{|c|}{ Varianza total explicada } \\
\hline & \multicolumn{3}{|c|}{ Autovalores iniciales } & \multicolumn{3}{|c|}{$\begin{array}{c}\text { Sumas de las saturaciones al cuadrado de la } \\
\text { extracción }\end{array}$} \\
\hline & Total & $\begin{array}{l}\text { \% de la } \\
\text { varianza }\end{array}$ & $\%$ acumulado & Total & $\begin{array}{l}\% \text { de la } \\
\text { varianza }\end{array}$ & $\%$ acumulado \\
\hline 1 & 9,307 & 35,796 & 35,796 & 9,307 & 35,796 & 35,796 \\
\hline 2 & 2,229 & 8,573 & 44,368 & 2,229 & 8,573 & 44,368 \\
\hline 3 & 1,880 & 7,229 & 51,598 & 1,880 & 7,229 & 51,598 \\
\hline 4 & 1,348 & 5,184 & 56,782 & 1,348 & 5,184 & 56,782 \\
\hline 5 & 1,232 & 4,740 & 61,522 & 1,232 & 4,740 & 61,522 \\
\hline 6 & 1,094 & 4,209 & 65,731 & 1,094 & 4,209 & 65,731 \\
\hline 7 & 1,008 & 3,877 & 69,608 & 1,008 & 3,877 & 69,608 \\
\hline 8 & .924 & 3,554 & 73,161 & & & \\
\hline 9 & 829 & 3,187 & 76,348 & & & \\
\hline 10 & .730 & 2,810 & 79,157 & & & \\
\hline
\end{tabular}

Como se observa los siete primeros factores explican en total el $69.6 \%$ de la variación total observada. Para una interpretación más sencilla de las cargas factoriales se programó una rotación ortogonal varimax. El objetivo fue tener un nuevo conjuntos de cargas vectoriales que sean ortogonales (independientes) entre sí, y que además maximicen la variancia explicada de cada factor. Como resultado se obtuvo el siguiente reporte: 
Matriz de componentes rotados ${ }^{a}$

\begin{tabular}{|l|l|l|l|l|l|l|l|}
\hline & \multicolumn{7}{|c|}{} \\
\cline { 2 - 7 } & 1 & 2 & 3 & 4 & 5 & 6 & 7 \\
\hline p1 & .116 & .782 & -.052 & .325 & .105 & -.087 & .182 \\
p2 & -.031 & .787 & .303 & .174 & -.033 & .143 & -.185 \\
p3 & .184 & .589 & .016 & .063 & .421 & .248 & .046 \\
p4 & .150 & .353 & .052 & .210 & .691 & .066 & .198 \\
p5 & .197 & .381 & .019 & .659 & .205 & .033 & .261 \\
p6 & .347 & .582 & .026 & .341 & -.019 & -.070 & .381 \\
p7 & .187 & .106 & .330 & .169 & .329 & .220 & .571 \\
p8 & .081 & .029 & .124 & .047 & .093 & .409 & .798 \\
p9 & .108 & .174 & .124 & .784 & .052 & .200 & .050 \\
p10 & .149 & .008 & .141 & .084 & -.008 & .855 & .279 \\
p11 & .281 & .041 & .149 & .240 & .176 & .773 & .141 \\
p12 & .102 & .064 & .759 & .148 & -.096 & .221 & .024 \\
p13 & .272 & .318 & .389 & .376 & .291 & .087 & -.008 \\
p14 & -.132 & .180 & .660 & -.039 & .277 & .008 & .209 \\
p15 & .748 & .166 & .001 & .211 & .112 & .231 & .089 \\
p16 & .753 & .049 & .029 & .195 & .252 & .299 & .013 \\
p17 & .375 & .103 & .347 & .439 & .508 & .042 & -.035 \\
p18 & .212 & -.120 & .381 & -.002 & .642 & -.015 & .099 \\
p19 & .379 & .431 & .241 & -.160 & .396 & -.097 & .022 \\
p20 & .460 & .409 & .314 & .120 & .281 & -.096 & .009 \\
p21 & .319 & -.005 & .637 & .193 & .287 & .047 & .084 \\
p22 & .569 & -.004 & .390 & .405 & -.019 & -.251 & .248 \\
p23 & .575 & .239 & .306 & .076 & .100 & .307 & .239 \\
p24 & .503 & .121 & .546 & -.076 & .147 & .155 & .078 \\
p25 & .353 & .284 & .121 & .504 & .305 & .269 & -.158 \\
p26 & .035 & .157 & -.005 & .463 & .594 & .273 & .091 \\
\hline
\end{tabular}

Método de extracción: Análisis de componentes principales. Método de rotación: Normalización Varimax con Kaiser.

a. La rotación ha convergido en 11 iteraciones.

Considerando que el número de encuestas fue de 119, se utilizó la una correlación de 0.5 como punto de discriminación de la asociación de las variables a cada uno de los factores. No se utilizó un valor de correlación más baja ya que es conveniente asociar cada variable a un único factor. Como se puede observar, el primer factor, que brinda una mayor explicación de la variabilidad total, se asocia a los factores:

p15 $=$ Idioma
p16 $=$ Diferencia cultural
p22 $=$ Fuerte competencia internacional
p23 $=$ Riesgo en la variación del tipo de cambio
p24 $=$ Alto valor de la moneda peruana (Nuevo sol)

El segundo factor, que conjuntamente al primero brindan un $44.4 \%$ de explicación de la variabilidad total, se asocia a los factores:

p1 $=$ Falta de conocimiento del potencial de mercados de exportación

p2 $=$ Falta de personal dedicado al planeamiento exportador

p3 = Falta de conocimiento de programas sobre asistencia exportadora

p6 $=$ Falta de información sobre las oportunidades para su producto / servicio en el extranjero 
Asociados al tercer factor se tiene:

p12= Altos costos de fletes $y$ transporte

p14= Documentación y trámites burocráticos requeridos para la operación de exportación

p21 = Dificultades logísticas

Asociados al cuarto factor se tiene:

p5 $=$ Falta general de conocimiento de cómo exportar

p9= Insuficiente capacidad de producción de la empresa

p25= Riesgo de pérdida de participación en el mercado local por la venta en el exterior

Asociados al quinto factor se tiene:

p4= Desconocimiento de los beneficios financieros y no financieros que la exportación puede generar

p17= Barreras arancelarias a las exportaciones

p18= Barreras no arancelarias relacionadas con la estandarización y certificaciones de los productos

p26= La inestabilidad política en el país de destino

Asociados al sexto factor se tiene:

p10 $=$ Falta de bancos locales con adecuada experiencia internacional

p11= Inadecuada red extranjera de los bancos con los que se opera

Asociados al sétimo factor se tiene:

p7 $=$ Elevados costos financieros en los métodos de pago utilizados en las operaciones internacionales

p8= Falta de financiamiento para afrontar el periodo de recupero de una inversión

Por otro lado los siguientes factores no parecen estar siendo explicados por ninguno de los componentes factoriales anteriores;

p19= Encontrar un distribuidor o canales de distribución adecuado

p20 $=$ El costo de adaptación del producto para el mercado extranjero

Además el factor p13= Diferencias en la utilización del producto en los mercados internacionales no se encuentra relacionado con ninguno de los factores.

Para la medición de las disimilitudes entre las percepciones sobre los factores que limitan la actividad exportadora se decidió considerar las distancias simples euclidianas entre las calificaciones otorgadas por los encuestados a cada una de los factores. Como en todos los casos las calificaciones corresponden a una escala Likert, no fue necesario realizar transformaciones de datos para evitar la influencia de las escalas de medida de las variables en la cuantificación de las medidas de disimilitud entre los factores. 
Considerando las respuestas recolectadas en 119 encuestas se obtiene una rápida convergencia a una solución que muestra un coeficiente $S$-stres de 0.00087 , muy cercano al óptimo deseado, lo que indica que el grado de bondad de ajuste es bueno.

El mapa perceptual obtenido es:

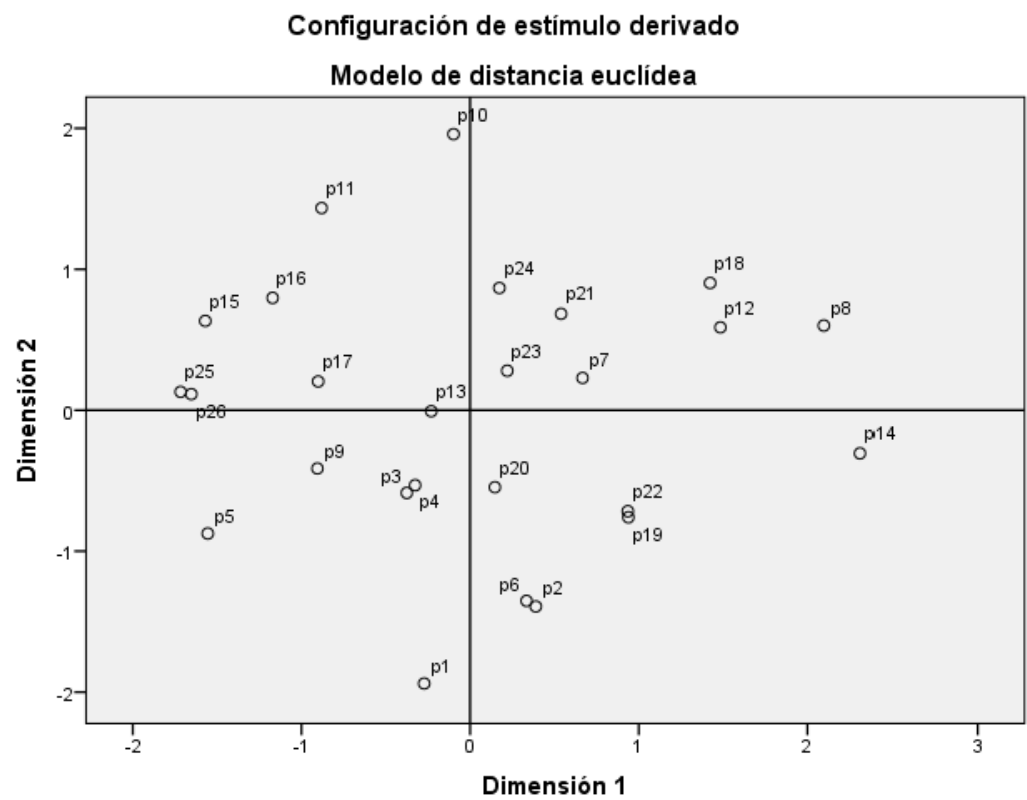

De acuerdo al gráfico anterior se puede deducir que hay ciertas analogías en las calificaciones de los factores, que conducen a la siguiente agrupación:

\begin{tabular}{|c|c|c|c|c|c|c|c|c|c|c|c|c|}
\hline \multirow[b]{2}{*}{ p01 } & \multirow[b]{2}{*}{ Falta de conocimiento del potencial de mercados de exportación } & \multicolumn{5}{|c|}{ Grupos } & \multicolumn{6}{|c|}{ Factores } \\
\hline & & G1 & & & & & & F2 & & & & \\
\hline p02 & Falta de personal dedicado al planeamiento exportador & G1 & & & & & & F2 & & & & \\
\hline p03 & Falta de conocimiento de programas sobre asistencia exportadora & & & & & G5 & & F2 & & & & \\
\hline p04 & Desconocimiento de los beneficios financieros y no financieros que la exportación puede generar & & & & & G5 & & & & $\mathrm{F}$ & & \\
\hline p05 & Falta general de conocimiento de cómo exportar & & & G3 & & & & & & F4 & & \\
\hline p06 & Falta de información sobre las oportunidades para su producto / servicio en el extranjero & G1 & & & & & & F2 & & & & \\
\hline p07 & Elevados costos financieros en los métodos de pago utilizados en las operaciones internacionales & & & & & G5 & & & & & & F7 \\
\hline p08 & Falta de financiamiento para afrontar el periodo de recupero de una inversión & & & & G4 & & & & & & & F7 \\
\hline p09 & Insuficiente capacidad de producción de la empresa & & & G3 & & & & & & F4 & & \\
\hline p10 & Falta de bancos locales con adecuada experiencia internacional & & G2 & & & & & & & & F6 & \\
\hline p11 & Inadecuada red extranjera de los bancos con los que se opera & & G2 & & & & & & & & F6 & \\
\hline p12 & Altos costos de fletes y transporte & & & & G4 & & & & F3 & & & \\
\hline p13 & Diferencias en la utilización del producto en los mercados internacionales & & & & & G5 & & & & & & \\
\hline p14 & Documentación y trámites burocráticos requeridos para la operación de exportación & & & & G4 & & & & F3 & & & \\
\hline p15 & Idioma & & & G3 & & & F1 & & & & & \\
\hline p16 & Diferencia cultural & & & G3 & & & F1 & & & & & \\
\hline p17 & Barreras arancelarias a las exportaciones & & & G3 & & & & & & & & \\
\hline p18 & Barreras no arancelarias relacionadas con la estandarización y certificaciones de los productos & & & & G4 & & & & & & & \\
\hline p19 & Encontrar un distribuidor o canales de distribución adecuado & G1 & & & & & & & & & & \\
\hline p20 & El costo de adaptación del producto para el mercado extranjero & & & & & G5 & & & & & & \\
\hline p21 & Dificultades logísticas & & & & & G5 & & & F3 & & & \\
\hline p22 & Fuerte competencia internacional & G1 & & & & & F1 & & & & & \\
\hline p23 & Riesgo en la variación del tipo de cambio & & & & & G5 & F1 & & & & & \\
\hline p24 & Alto valor de la moneda peruana (Nuevo sol) & & & & & G5 & F1 & & & & & \\
\hline p25 & Riesgo de pérdida de participación en el mercado local por la venta en el exterior & & & G3 & & & & & & $\mathrm{F} 4$ & & \\
\hline p26 & La inestabilidad política en el país de destino & & & G3 & & & & & & & & \\
\hline
\end{tabular}


Por otro lado los siguientes factores no parecen estar siendo explicados por ninguno de los componentes factoriales anteriores: p19 (encontrar un distribuidor o canales de distribución adecuado), p20 (el costo de adaptación del producto para el mercado extranjero).

Considerando que el objetivo del análisis factorial es encontrar la factores que resultan ser más influyentes en la actividad exportadora, se han considerado solamente los tres primeros factores ya que ellos explican en conjunto un $51.6 \%$ de la variación total. La adición de otros factores no incrementa significativamente la variación explicada, y por otro lado incluye a demasiados factores imposibilitando hacer una precisión sobre cuáles son los de mayor importancia.

\section{Las barreras asociadas al primer factor son:}

\begin{tabular}{|c|c|c|c|}
\hline & Indicador & Clasificación general & \begin{tabular}{|ll}
\multicolumn{3}{|l}{ Clasificación } \\
de & la \\
encuesta &
\end{tabular} \\
\hline $\mathrm{p} 15=$ & Idioma & $\begin{array}{l}\text { Externa, Mercado de } \\
\text { destino. Sociocultural }\end{array}$ & $\begin{array}{l}\text { Barrera de } \\
\text { procedimiento }\end{array}$ \\
\hline $\mathrm{p} 16=$ & Diferencia cultural & $\begin{array}{l}\begin{array}{l}\text { Externa, Mercado de } \\
\text { destino. Sociocultural }\end{array} \\
\end{array}$ & $\begin{array}{l}\text { Barrera de } \\
\text { procedimiento }\end{array}$ \\
\hline $\mathrm{p} 22=$ & Fuerte competencia internacional & $\begin{array}{l}\text { Externa, Mercado de } \\
\text { destino. Obstáculos al } \\
\text { desarrollo de mercados }\end{array}$ & $\begin{array}{l}\text { Barrera } \\
\text { Exógena }\end{array}$ \\
\hline p23= & $\begin{array}{l}\text { Riesgo en la variación del tipo de } \\
\text { cambio }\end{array}$ & $\begin{array}{l}\text { Externa. Mercado de } \\
\text { origen. Riesgos y retorno }\end{array}$ & $\begin{array}{l}\text { Barrera } \\
\text { Exógena }\end{array}$ \\
\hline p24= & $\begin{array}{l}\text { Alto valor de la moneda peruana } \\
\text { (Nuevo sol) }\end{array}$ & $\begin{array}{l}\text { Externa. Mercado de } \\
\text { origen. Riesgos y retorno }\end{array}$ & $\begin{array}{l}\text { Barrera } \\
\text { Exógena }\end{array}$ \\
\hline
\end{tabular}

Asociados al segundo factor se tiene:

\begin{tabular}{|c|c|c|c|}
\hline & Indicador & Clasificación general & \begin{tabular}{|l|}
\multicolumn{2}{|l}{ Clasificación } \\
de la \\
encuesta
\end{tabular} \\
\hline $\mathrm{p} 1=$ & $\begin{array}{l}\text { Falta de conocimiento del potencial de } \\
\text { mercados de exportación }\end{array}$ & $\begin{array}{l}\text { Interno. Conocimiento y } \\
\text { experiencia }\end{array}$ & $\begin{array}{l}\text { Barrera de } \\
\text { conocimiento }\end{array}$ \\
\hline $\mathrm{p} 2=$ & $\begin{array}{l}\text { Falta de personal dedicado al } \\
\text { planeamiento exportador }\end{array}$ & \begin{tabular}{|l|l|}
$\begin{array}{l}\text { Interno. Enfoque } \\
\text { compromiso gerencial }\end{array}$ & \\
\end{tabular} & \begin{tabular}{|l|} 
Barrera de \\
conocimiento
\end{tabular} \\
\hline $\mathrm{p} 3=$ & $\begin{array}{l}\text { Falta de conocimiento de programas } \\
\text { sobre asistencia exportadora }\end{array}$ & \begin{tabular}{|l}
$\begin{array}{l}\text { Externo. Mercado } \\
\text { origen y y política } \\
\text { gobierno }\end{array}$ \\
\end{tabular} & $\begin{array}{l}\text { Barrera de } \\
\text { conocimiento }\end{array}$ \\
\hline$p 6=$ & $\begin{array}{l}\text { Falta de información sobre las } \\
\text { oportunidades para su producto / } \\
\text { servicio en el extranjero. }\end{array}$ & Interno. Información & $\begin{array}{l}\text { Barrera de } \\
\text { conocimiento }\end{array}$ \\
\hline
\end{tabular}

Asociados al tercer factor se tiene:

\begin{tabular}{|c|c|c|c|}
\hline & Indicador & Clasificación general & $\begin{array}{l}\text { Clasificación } \\
\text { de la } \\
\text { encuesta }\end{array}$ \\
\hline $\mathrm{p} 12=$ & Altos costos de fletes y transporte & $\begin{array}{l}\text { Externo. Obstáculos al } \\
\text { desarrollo de mercados }\end{array}$ & $\begin{array}{l}\text { Barrera de } \\
\text { procedimiento }\end{array}$ \\
\hline $\mathrm{p} 14=$ & $\begin{array}{l}\text { Documentación y } \\
\text { burocráticos requeridos para la la } \\
\text { operación de exportación }\end{array}$ & $\begin{array}{lll}\begin{array}{l}\text { Externo. } \\
\text { origen. }\end{array} & \text { Políticas } & \text { de } \\
\text { gobierno. } & & \end{array}$ & $\begin{array}{l}\text { Barrera de } \\
\text { procedimiento }\end{array}$ \\
\hline $\mathrm{p} 21=$ & Dificultades logísticas & \begin{tabular}{|lll} 
Interno. & Marketing $\quad \mathrm{y}$ \\
logística & &
\end{tabular} & $\begin{array}{l}\text { Barrera de } \\
\text { procedimiento }\end{array}$ \\
\hline
\end{tabular}

\section{Conclusiones}

Los resultados obtenidos en el presente estudio, son una referencia a tener en cuenta para revisar la política de promoción de exportaciones, más aun tratándose de empresas esporádicas las mismas que de contar con apoyo ad-hoc podrían mejorar su 
desempeño y convertirse en exportadoras, contribuyendo de esta manera a incrementar el número de empresas exportadoras. Por otro lado, los resultados encontrados proporcionan a las empresas pautas a tener en cuenta para mejorar sus capacidades con el objeto de orientarse hacia los mercados internacionales.

La literatura revisada muestra una gran escasez de trabajos en países de América Latina, con la excepción de Brasil, el cual cuenta con tres (3) trabajos que analizan los obstáculos a la exportación. Los países desarrollados son los que generalmente cuentan con la mayor cantidad de estudios, siendo Estados Unidos e Inglaterra los que lideran la lista de estudios.

Los obstáculos que limitan la expansión de las empresas en los mercados internacionales, se clasifican como: internas y externas. Las variables de cada una de ellas son diversas y su importancia está en función a la percepción que cada empresa tenga de cada variable.

Las variables internas se subdividen en: (a) de enfoque y compromiso gerencial; (b) de conocimiento y experiencia; (c) de información, funcional y de marketing.

Las variables externas se subdividen en: (a) de mercado de destino y (b) de origen.

De la revisión de la literatura, se ha preparado un cuadro el mismo que muestra cincuenta y siete (57) variables y ochenta (80) autores. (Ver el resumen en el cuadro de Excel que se adjunta). En el proceso de la revisión de la literatura, se encontraron estudios de meta-análisis como los de Tinashe, (2013), Tesfom \& Lutz, (2006), Leonidou L. C.,(1995 - B) Leonidou L. C.,( 2004) que sirvieron de base para desarrollar el cuadro de los autores y variables.

En el caso del Perú y luego de revisar los resultados, no se encontró ninguna barrera adicional en común, y las respuestas más bien estuvieron dirigidas a problemas específicos de la empresa y en muchos casos, repetían en otros términos las barreras propuestas, calificándolas con mayor severidad que la barrera original.

Los resultados obtenidos con los métodos de análisis factorial y de escalamiento multidimensional presentan grandes semejanzas, con algunas barreras que muestran una gran influencia sobre la actividad exportadora. Dichas semejanzas se aprecian en los resultados obtenidos con los tres primeros factores del análisis factorial en donde se observa que sus cargas factoriales muestran una gran diferencia con las cargas de otras barreras que no son tan relevantes en la actividad exportadora.

Si bien los tres primeros factores alcanzan un porcentaje de $51.6 \%$ de explicación, se observa que los siguientes cuatro factores explican en conjunto un $18 \%$ de la variación total, lo que indica que existen otras barrera que deben ser analizadas en un estudio más detallado, sobre todo evaluando su influencia en cada segmento de empresas exportadoras (esporádicas y continuas), así como al sector al cual pertenezcan.

De acuerdo a la clasificación de las barreras u obstáculos empleada en la encuesta, encontramos que el primer factor, el más importante, está asociado principalmente a barreras de procedimiento y barreras exógenas, y de acuerdo a la clasificación general corresponden a las barreras externas, tanto del mercado de destino como en origen. 
El segundo factor, está asociado principalmente a las barreras de conocimiento, y de acuerdo a la clasificación general corresponden a las barreras de carácter interno.

El tercer factor, está asociado principalmente a barreras de procedimiento, y de acuerdo a la clasificación general corresponden a las barreras externas asociadas a obstáculos de desarrollo del mercado, mercado de origen, políticas de gobierno, e internas asociados a marketing y logística.

La variables agrupadas en el primer factor muestran en primer lugar la necesidad de reforzar las capacidades gerenciales de las empresas, dado que en un mundo globalizado con distancias cada vez más cortas como consecuencia del desarrollo de las tecnologías de información que hacen necesario que los empresarios tengan competencias idiomáticas así como habilidades para poder desenvolverse en mercados con alta diversidad cultural. Tanto el idioma como la diferencia cultural, si bien es cierto son variables externas a la empresa, o son parte de los procedimientos que deben conocerse para poder acceder y mantenerse en los mercados, son necesarios y condición para competir internacionalmente. Del mismo modo, en este factor se indica como variables importantes la competencia internacional y las variaciones del tipo de cambio, lo cual indican la gran necesidad de saber diseñar y gestionar las estrategias más adecuadas para la exportación, dado que éstas variables son típicas de los mercado internacionales que usualmente son identificadas y analizadas tanto en la formulación del plan como en la estrategia de exportación.

Respecto a las variables asociada con el segundo factor, éstas son claras en mostrar como barreras la falta de experiencia y conocimiento relacionados a la gestión de la comercialización internacional, dado que en este tópico es usual conocer las técnicas de identificación y dimensionamiento de los mercados de exportación así como el utilizar las diversas fuentes de información como las bases de datos especializadas en comercio internacional que facilitan el diseño y la gestión estratégica de la empresa. Adicionalmente, las variables indicadas muestran como una debilidad estructural que están relacionadas tanto al primer factor como al segundo, la falta de personal dedicado al planeamiento exportador, lo cual explica de manera clara las barreras percibidas por la empresa y la necesidad de especializar recursos humanos en el ámbito de las exportaciones y de la internacionalización de la empresa.

El tercer factor está constituido por variables externas a la empresa y tiene que ver todas con la logística de exportación, que está constituido por procesos especializados y demandan recursos humanos especializados que conozcan tanto de la gestión en estos temas como de implementar políticas que el estado debe desarrollar para disminuir el impacto negativo de estas variables en el desempeño de las empresas.

El Estado, a través de los organismos competentes, debe reforzar tanto el diseño como la gestión de las políticas de promoción de exportación, a efecto de superar la tercera variable del segundo factor, la cual muestra de manera muy clara que las empresas esporádicas no cuentan con suficiente información de los programas que desarrollan. 


\section{Referencias bibliográficas}

Abassi, M., Shakhsian, F., Fayyazi, M., \& Rezaie, M. (2012). Identifying the Most Important Export Barriers in Iran Case Study: Auto-Part Industry. International Journal of Academic Research in Economics and Management Sciences. January 2012, Vol. 1, No. 1, 63-81.

Acedo, F. J., \& Casillas, J. C. (2005). Current paradigms in the international management field: An author co-citation analysis. International Business Review(14), 619-639.

Aharoni, A. N. D. (1966). The Foreign Investment Decision Process, Harvard Graduate School of Business administration, Division of Research, Boston.

Alexandrides, C. G. (1971). How the major obstacles to exporting can be overcome, Atlantic Economic Review, May, pp. 12-15.

Amit, R., \& Schoernaker, P. J. (1993). Strategic Assets and Organizational Rent. Strategic Management Journal 14(1), 33-46.

Anderson , T. (1972). An Intruction to Multivariate Statistical Analysis. New York: Johon Wiley \& Sons.

Anderson, H. (1999). Análisis Multivariante. Madrid-España: Prentice-Hall.

Artega-Ortiz, J., \& Fernandez-Ortiz, R. (2010). Why Don't We Use the Same Export Barrier Measurement Scale? An Empirical Analysis in Small and Medium-Sized Enterprises. Journal of Small Business Management 2010 48(3), 395-420.

Barney, J. (1991). Firm Resources ans Sustained Competitive Advantage. Journal of Management 17(1), 99-120.

Barret, N. I. and Wilkinson, I. F. (1985), "Export stimulation: a segmentation study of the exporting problems of Australian manufacturing firms", European Journal of Marketing, Vol. 19 No. 2, pp. 53-72.

Bauerschmidt, A., Sullivan, D., \& Gillespie, K. (1985). Common Factors Underlying Barriers to Export: Studies in the U. S. Paper Industry. Journal of International Business Studies, Vol. 16, No. 3 (Autumn), 111-123.

Bauerschmidt, A., Sullivan, D., \& Gillespie, K. (1985). Common factors underlying barriers to export: studies in the US paper industry. Journal of International Business Studies, 16(3), 11-23.

Bilkey, W. (1978). An Attempted Integration of the Literature on the Ex port Behavior of Firms. Journal of International Business Studies , 33-46.

BILKEY, W. J., \& TESAR, G. (1977). The Export Behavior of Smaller-Sized Wisconsin Manufacturing Firms. Journal of International Business Studies, 93-98.

Boddewyn, J. (1999). The domain of international management. Journal of International Management(5), 3-14. 
Bodur, M. (1986). A study on the nature and intensity of problems experienced by Turkish exporting firms. Advances in International Marketing, 1, 32 - 205.

Cateora, P. and Graham, J. (2001).International MarketingUSA: Irwin/McGraw-Hill.

Cavusgil, S., \& Nevin, J. (1981). Internal Determinants of Export Marketing Behavior: An Empirical Investigation. Journal of Marketing Research, Vol. 18, No. 1, 114-119.

Chan, K. C., Fung, H. G., \& Leung, W. K. (2006). International business research: trends and school rankings. International Business Review(15), 317-338.

Christensen, C., Da Rocha, A., \& Gertner, R. K. (1987). An empirical investigation of factores influencing exporting success of Brazilian firms. The Journal of International Business Studies, 18(3), 61-77.

Constantine Katsikeas, Morgan Robert. (1994). Differences in perceptions of exporting problems based on firm size and export market experience. European Journal of Marketing, 17-35.

Craig, J. (2003). Export Marketing Performance:A Study of Thailand Firms. Journal of Small Business Management, 213-221.

Craig, J., \& Zafar, A. (2005). The impact of barriers to export on export marketing performance. Journal of Global Marketing, 19(1), 71-94.

Cuadras, C. (2012). Nuevos Métodos de Análisis Multivariante. Barcelona-España: CMC Editions.

Cuervo-Cazurra, A., Maloney, M. M., \& Manrakhan, S. (2007). Causes of the Difficulties in Internationalization. Journal of International Business Studies 38(5), 709125.

Czinkota, \& Michael, R. (2011). Administración de la Mercadotecnica. México D.F: Thomson Learning.

Da Silva, P., \& Da Rocha, A. (2001). Perception of export barriers to Mercosur by Brazilian firms. International Marketing Review, 18(6), 589-610.

Dichtl, E., KoÈglmayr, H. G., \& Muller , S. (1986). Identifying export potential: a comparative analysis of German and Japanese firms. Advances in International Marketing, 1, 233-54.

Doole, Isobel and Robin Lowe (2001). International Marketing Strategy. UK: Thomson Learning.

Dubois , F. L., \& Reeb, D. (2000). Ranking the international business journals. Journal of International Business Studies(31(4)), 689-704.

Eriksson, K., Johanson, J., Majkgard, A., \& Sharma, D. D. (1997). Experimental Knowledge and Costs in the Internationalization Process. Journal of International Business Studies 28(2), 337-360. 
Gómez-Mejía, L. (1988). The Role of Human Resources Strategy in Export Performance: A Longitudinal Study, Strategic Management Journal 9, 493-505.

Gomez-Mejia, L. R., \& Balkin, D. (1992). Determinants of faculty pay: An Agency Theory perspective. Academy of Management Journal(35), 921-955.

Gripsrud, G. (1990). The determinants of export decisions and attitudes to a distant marketi: Norwegian fishery exports to Japan. Journal of International Business Studies, 21(3), 85-469.

Hakan , M., \& Tokol, T. (2007). The effects of export barriers on perceived export performance: An empirical research on SMEs in Turkey. EuroMed Journal of Business, 2(1), 36 - 56.

Hastie T., T. T. (2008). Data Mining, Inference, and Prediction. Stanford-California: Springer.

Johanson, J., \& Vahlne, J. E. (1977). The Internationalization Process of the Firm: A Model of Knowledge Development and Increasing Foreign Market Commitments. Journal of International Business Studies 8(1), 23-32.

Johanson, J., \& Wiedersheim-Paul, F. (1975). The Internationalization of the Firm: Four Swedish Case Studies. Journal of Management Studies 12(3), 305-322.

Johnson, D. (2000). Métodos Multivariados Aplicados al Análisis de datos. México: Thomson.

Kaleka, A., \& Katsikeas, C. (1995). Exporting Problems: The Relevance of Export Development. Journal of Marketing Management, 11(5), 499-515.

Kamath, Shyam, Philip J. Rosson, Donald Patton, and Mary Brooks (1987). Research on Success in Exporting: Past, Present and Future, in Managing Export Entry and Expansion. Ed. P. J. Rosson and S. D. Reid. New York: Praeger Publishers, 398-421.

Katsikeas, C. (1994). Perceived Export Problems and Export Involvement: The Case of Greek Exporting Manufacturers. Journal of Global Marketing 7(4) , 29-57.

Kaynak, E., \& Barker, A. (1992). An Empirical Investigation of the Differences between Initiating and Continuing Exporters. European Journal of Marketing, 26(3), 27 - 36.

Kaynak, E. and Kothari, V. (1983), "Export behavior of small manufactures: a comparative study of American and Canadian firms", European Management Journal, Vol. 2 No. 2, Summer, pp. 41-7.

Kedia, B. and Chhokar, J. (1986). Factor Inhibiting Export Performance of Firms: An Empirical Investigation, Management International Review 26(4), 33-43.

Keng, Kau Ah, and Tan Soo Jiuan (1989), "Differences between Small and MediumSized Exporting and Nonexporting Firms: Nature and Nurture", International Marketing Review 6(4), 27-40. 
Kinnear T., T. J. (1996). Investigación de Mercados. Un enfoque aplicado. México: Mc Graw-Hill.

Korth, C. (1991). Managerial barriers to US exports . Business Horizons.

Kotabe, M., \& Czinkota, M. (1992). State government promotion of manufacturing exports: A gap analysis. Journal of International Business Studies, 23(4), 637-658.

Ledolter, J. (2013). Data Mining and Business Analytics with R. New Jersey: John Wiley \& Sons.

Lehman, D. (1997). Investigación y Análisis de Mercado". Ed. CECSA. 1997. México: CECSA.

Leonidou, L. (1995). Empirical Research on Export Barriers: Review, Assessment, and Synthesis. Journal of International Marketing, 3(1), 29-43.

Leonidou, L. C. (1995b). Empirical Research on Export Barriers:Review, Assessment, and Synthesis. Journal of International Marketing, 3(1), 29-43.

Leonidou, L. C. (1995). Export Barriers: Non-exporters' Perceptions. International Marketing Review, 4-25.

Leonidou, L. C. (1995). Export Barriers: Non-exporters' Perceptions. International Marketing Review, 4-25.

Leonidou, L. C. (2004). An Analysis of the Barriers Hindering Small Business Export Development . Journal os Small Business Management 42(3), 279-302.

Leonidou, L. C., Katsikeas, C. S., \& Piercy, N. G. (1998). Identifying Managerial Influences on Exporting: Past Research and Future Directions. Journal of International Marketing 6(2), 74-102.

Lu, J. W. (2003). The evolving contributions in international strategic management research. Journal of International Management (9), 193-213.

Malca, O. \& Rubio J. (2013). Continuidad y desempeño exportador en la empresa peruana. Journal of Business, Vol. 5(1): 52-75.

Martinez, Z., \& Toyne, B. (2000). What is international management, and what is its domain? Journal of International Management(6), 11-28.

McConnel, J.E. (1979). The Export Decision: An Empirical Study of the Firm Behavior. Economic Geography, Vol. 55 No. 3, July, pp. 171-83.

Milanzi, M. (2012). The Impact of Barriers on Export Behavior of a Developing Country Firms: Evidence from Tanzania. International Journal of Business and Management Vol. 7, No. 3; February 2012.

Morgan, R., \& Katsikeas, C. (1997). Obstacles to Export Initiation and Expansion. Omega 25(6), 677-690. 
Moini, H. A. (1997). Barriers Inhibiting Export Performance of Small and Medium-Sized Manufacturing Firms, Journal of Global Marketing 10(4), 67-93.

Pagano, R. (1999). Estadística para las Ciencias del Comportamiento. México: Thomson.

Pinho, C., \& Martins, L. (2010). Exporting barriers: insights from Portuguese small-and medium- sized. Journal of International Entrepreneurship, 254-272.

Pisani, N. (2011). International management research: Investigating its recent diffusion in top management journal. Journal of Management(35(2)), 199-218.

Rabino, S. (1980). An examination of barriers to exporting encountered by small manufacturing companies. Management International Review, 20(1), 67-73.

Ravi, S., \& Terpstra , V. (1997). International marketing. Fort Worth The Dryden Press .

Roth, K., Kostova, T., \& Dakhli, M. (2011). Exploring cultural misfit: Causes and consequences. International Business Review(20), 15-26.

Terpstra Vern, and Ravi Sarathy (2000). International Marketing. USA: Dryden Press.

Tesar, G. and Jesse S. (1982), "Comparison of Winsconsin and Virginian Small and Medium-Sized Exporters: Aggressive and Passive Exporters", in Export Management: An International Context. Ed. M. R. Czinkota and G. Tesar. New York: Praeger Publishers, 85-112.

Tesfom, G., \& Lutz, C. (2006). A classification of export marketing problems of small and medium sized manufacturing firms in developing countries. International Journal of Emerging, 1(3), 262-281.

Tesfom, G., \& Lutz, C. (2006). A classification of export marketing problems of small and medium sized manufacturing firms in developing countries. International Journal of Emerging markets, 262-281.

Tinashe, E. (2013). Export Barriers and Path to Internationalization: A Comparison of Conventional Enterprises and International New Ventures. Journal of International Entrepreneurship, 3-29.

Vozikis, G. and Mescon, T. (1985). "Small Exporters and Stages of Development: And Empirical Study", American Journal of Small Business (Summer), 49-64.

Werner, S. (2002). Recent development in international management research: A review of 20 top management journals. Journal of Management(28(3)), 277-305.

Werner, S., \& Brouthers, L. E. (2002). How international is management . Journal of International Business Studies, 33(3), 583-591.

Yaprak, Attila (1985). "An Empirical Study of the Differences between Small Exporting and Nonexporting U.S. Firms", International Marketing Review 2(2), 72-83. 
ANEXO 1: Encuesta. Sobre la base de Artega-Ortiz \& Fernandez-Ortiz, (2010)

Identificación y análisis de los obstáculos que limitan la actividad exportadora

Llene los datos que se les solicita a continuación.

\begin{tabular}{|l|l|}
\hline \multicolumn{2}{|l|}{ Datos de control } \\
\hline D1 & Nombre del entrevistado \\
\cline { 2 - 2 } D2 & Cargo dentro de la empresa \\
\cline { 2 - 2 } D3 & Fecha de nacimiento \\
D4 & Formación \\
\cline { 2 - 2 } D5 & Correo electrónico \\
D6 & Teléfono \\
\cline { 2 - 2 } D7 & Nombre de la empresa \\
D8 & Actividad de la empresa \\
\hline
\end{tabular}

¿En qué medida cada una de las siguientes barreras a la exportación hace difícil iniciar o ampliar su actividad exportadora? Siendo 1 "no dificulta" y 7 "dificulta enormemente".

\begin{tabular}{|c|c|c|c|c|c|c|c|c|}
\hline \multicolumn{2}{|r|}{ Barreras a la exportación } & \multicolumn{7}{|c|}{ Grado de dificultad } \\
\hline B1 & Falta de conocimiento del potencial de mercados de exportación & 1 & 2 & 3 & 4 & 5 & 6 & 7 \\
\hline B2 & Falta de personal dedicado al planeamiento exportador & 1 & 2 & 3 & 4 & 5 & 6 & \\
\hline B3 & Falta de conocimiento de programas sobre asistencia exportadora & 1 & 2 & 3 & 4 & 5 & 6 & 7 \\
\hline B4 & $\begin{array}{l}\text { Desconocimiento de los beneficios financieros y no financieros que la } \\
\text { exportación puede generar. }\end{array}$ & 1 & 2 & 3 & 4 & 5 & 6 & 7 \\
\hline B5 & Falta general de conocimiento de cómo exportar & 1 & 2 & 3 & 4 & 5 & 6 & 7 \\
\hline B6 & $\begin{array}{l}\text { Falta de información sobre las oportunidades para su producto / servicio en } \\
\text { el extranjero. }\end{array}$ & 1 & 2 & 3 & 4 & 5 & 6 & 7 \\
\hline B7 & $\begin{array}{l}\text { Elevados costos financieros en los métodos de pago utilizados en las } \\
\text { operaciones internacionales. }\end{array}$ & 1 & 2 & 3 & 4 & 5 & 6 & 7 \\
\hline B8 & $\begin{array}{l}\text { Falta de financiamiento para afrontar el periodo de recupero de una } \\
\text { inversión. }\end{array}$ & 1 & 2 & 3 & 4 & 5 & 6 & 7 \\
\hline B9 & Insuficiente capacidad de producción de la empresa & 1 & 2 & 3 & 4 & 5 & 6 & 7 \\
\hline B10 & adecuada experiencia internacional & 1 & 2 & 3 & 4 & 5 & 6 & 7 \\
\hline B11 & tranjera de los bancos con los que se opera & 1 & 2 & 3 & 4 & 5 & 6 & 7 \\
\hline B12 & os de fletes y transporte & 1 & 2 & 3 & 4 & 5 & 6 & 7 \\
\hline B13 & Diferencias en la utilización del producto en los mercados internacionales & 1 & 2 & 3 & 4 & 5 & 6 & 7 \\
\hline B14 & $\begin{array}{l}\text { Documentación y trámites burocráticos requeridos para la operación de } \\
\text { exportación }\end{array}$ & 1 & 2 & 3 & 4 & 5 & 6 & 7 \\
\hline B15 & Idioma & 1 & 2 & 3 & 4 & 5 & 6 & 7 \\
\hline B16 & Difere & 1 & 2 & 3 & 4 & 5 & 6 & 7 \\
\hline B17 & Barreras arancelarias a & 1 & 2 & 3 & 4 & 5 & 6 & 7 \\
\hline B18 & $\begin{array}{l}\text { certificaciones de los productos (Ej: requisitos fitosanitarios o barreras } \\
\text { similares) }\end{array}$ & 1 & 2 & 3 & 4 & 5 & 6 & 7 \\
\hline B19 & Encontrar un distribuidor o canales de distribución adecuado & 1 & 2 & 3 & 4 & 5 & 6 & 7 \\
\hline B20 & ación del producto para el mercado extranjero & 1 & 2 & 3 & 4 & 5 & 6 & 7 \\
\hline B21 & Dificultade & 1 & 2 & 3 & 4 & 5 & 6 & 7 \\
\hline B22 & Fuerte competencia inter & 1 & 2 & 3 & 4 & 5 & 6 & 7 \\
\hline B23 & Riesgo en la variación del t & 1 & 2 & 3 & 4 & 5 & 6 & 7 \\
\hline B24 & Alto valor de la moneda peruana (Nuevo sol) & 1 & 2 & 3 & 4 & 5 & 6 & 7 \\
\hline B25 & $\begin{array}{l}\text { Riesgo de pérdida de participación en el mercado local por la venta en el } \\
\text { exterior }\end{array}$ & 1 & 2 & 3 & 4 & 5 & 6 & 7 \\
\hline B26 & La inestabilidad política en el país de destino & 1 & 2 & 3 & 4 & 5 & 6 & 7 \\
\hline B27 & $\begin{array}{l}\text { Considerar los siguientes casilleros si existen otras barreras a la exportación } \\
\text { que no han sido incluidas, por favor indique cuál es, y califiquela con un } \\
\text { puntaje entre } 1 \text { y } 7 \text {. }\end{array}$ & & & & & & & \\
\hline B28 & $\begin{array}{l}\text { Considerar los siguientes casilleros si existen otras barreras a la exportación } \\
\text { que no han sido incluidas, por favor indique cuál es, y califiquela con un } \\
\text { puntaje entre } 1 \text { y } 7 \text {. }\end{array}$ & & & & & & & \\
\hline B29 & Grado total de dificultad & 1 & 2 & 3 & 4 & 5 & 6 & 7 \\
\hline
\end{tabular}

